SLAC-PUB-8094

March 1999

\title{
Report on the Tau-Charm Physics Workshop
}

\author{
Martin L. Perl and Peter C. Kim
}

Summary of theTau-Charm Physics Workshop,

Stanford, CA, USA, 3/6/99-3/9/99

Stanford Linear Accelerator Center, Stanford University, Stanford, CA 94309

Work supported by Department of Energy contract DE AC03 76SF00515. 
SLAC-PUB-8094

March 6-9, 1999

\section{REPORT ON THE TAU-CHARM PHYSICS WORKSHOP* March 6-9, 1999}

Martin L. Perl and Peter C. Kim Stanford Linear Accelerator Center Stanford, CA 94309, U. S. A.

March 26, 1999

\section{Table of Contents}

\section{EXECUTIVE SUMMARY}

\section{REPORT}

1. Introduction: The Tau-Charm Factory Concept and Tau-Charm Physics

2. Collider and Detector Design Status

3. Evaluation Criteria for Physics Capability of a Tau-Charm Factory

4. Glueball and Other Strong Interaction Physics

5. Tau Lepton Physics

6. D Meson Physics

7. Role of a Tau-Charm Factory in 21st Century Elementary Particle Physics Conclusions

APPENDIX : Workshop Participants

* Work supported by DOE contract DE-AC03-76SF00515 


\section{EXECUTIVE SUMMARY}

Physics research in the mass region 3 to $5 \mathrm{GeV}$ has been extraordinarily productive. Major discoveries there have been the tau lepton and the bare states of the charmed quarks. The region encompasses a rich spectroscopy of charm - anti-charm states and states of charm combined with lighter components. Exploration of this important physics is far from complete. Currently the only facility operating in a dedicated mode in the $3-5 \mathrm{GeV}$ mass region is the BEPC facility in Beijing. Other facilities operating in higher mass regions have also obtained results relevant to the $3-5 \mathrm{GeV}$ range as a by product of their work and will continue to do so. Examples are CESR, now operating for some time, and the asymmetric B-Factories about to come into operation as well as LEP and fixed target facilities.

This Workshop was designed to assess the results accumulated so far in the $3-5 \mathrm{GeV}$ range and to address the question whether a new, state of the art $\mathrm{e}^{+} \mathrm{e}^{-}$collider of $10^{33} \mathrm{~cm}^{-2} \mathrm{~s}^{-1}$ luminosity, operating in the mass range $3-5 \mathrm{GeV}$ with polarized and monochromatized beam capability, has sufficient merit to warrant construction. Such a facility has been referred to frequently as a "Tau-Charm-Factory". As seen at the Workshop, a more appropriate designation would be a "Frontier Facility for Tau, Charm, Light Quark and Gluon Physics".

The Workshop comprised a small group of experts from North America, China and Western Europe gathered March 6 - 9 with four goals:

a) to review the present understanding of tau, charm, light quark and gluon physics;

b) to stimulate new ideas and experimental direction in tau, charm, light quark and gluon physics;

c) to summarize what we might learn about these sub-atomic particles and their interactions from a dedicated frontier facility, such as a Tau Charm Factory operating in the 3-5 GeV region or a BFactory or other experimental facility; and

d) consider the role, advantages and disadvantages of using a Tau Charm Factory as compared to a B - Factory facility or other accelerator facility.

The Workshop study concludes that:

1.) Progress in the construction and successful initial operation of B-Factories has made us fully confident that building the collider and detector for a Tau-Charm Factory is feasible. The 
designers and builders of a Tau-Charm Factory will be able to continually learn from the experience and knowledge gained at B-Factories and at the newly constructed DAPHNE PhiFactory, an electron-positron collider facility operating in the $1 \mathrm{GeV}$ energy range.

2.) The luminosity of a Tau Charm Factory is expected to be two orders of magnitude larger than that of the BEPC at its maximum upgrade potential.

3.) A Tau-Charm Factory is a unique facility for carrying out broad and significant research in gluon and light-quark physics.

4.) With respect to gluonic matter and the existence of glueballs, a Tau-Charm Factory offers a unique opportunity for a very important significant physics result.

5.) While tau and charm physics will continue to be studied at other facilities such BFactories, a Tau-Charm Factory provides competitive, and in some cases special, experimental conditions for carrying out significant physics in many areas of tau and charm physics.

6.) These special experimental conditions at a Tau-Charm Factory include: the use of the tau energy threshold for tau physics studies, the use of the $\psi^{\prime \prime}$ resonance for the study of D meson physics, and the naturalness of including a polarized beam facility in the initial construction of the collider.

7.) The breadth of physics and the range of special experimental conditions inherent in a Tau-Charm Factory facility offer the possibility of major discoveries in areas such as CP violation and the lepton flavor non-conservation.

Above all:

8.) We look forward to exploring at the beginning of the 21 st Century those large parts of particle physics that we do not understand; therefore maximum range and variety in our experimental tools is crucial. A Tau-Charm Factory facility is different from a B-Factory facility or any other high energy physics facility. Therefore it amplifies the range and variety of experimental tools at a relatively moderate cost. 


\section{REPORT}

\section{Introduction}

Research in the energy region between 3-5 GeV has been extraordinarily productive and among other discoveries has revealed the existence of the tau lepton and of the bound and bare states of the charmed quarks. The region encompasses a rich spectroscopy of charm-anticharm states and the states of the charmed quark combined with lighter components. Exploration of this interesting and important physics has not even been remotely exhausted in the past and the only machine in the world operating in a dedicated mode in this energy region is the BEPC collider at the Institute of High Energy Physics in China. Results relevant to this energy range have been, and continue to be, accumulated by many high energy physics colliders in the world as a byproduct of their activities which emphasize other energy regions.

This workshop was designed to address the status of the physics results which have accumulated in the 3-5 GeV range of energy and to explore the question whether it warrants a new collider to be constructed, specifically dedicated to this energy region but with highly superior performance as to luminosity (specifically $10^{33} \mathrm{~cm}^{-2} \mathrm{sec}^{-1}$ ) and incorporating additional parameters, such as the possibility of polarized circulating beams or specialized optics providing highly monochromatic beams. Such a dedicated machine is called a Tau Charm Factory.

The Tau-Charm Factory concept was developed some years ago. A Tau-Charm Factory consists of a high luminosity electron-positron collider and associated high sensitivity and high resolution particle detector. The object of this proposed facility is to study the physics of the broad range of subatomic particles that are produced in the 3-5 GeV energy range. These particles include:

- the tau lepton;

- the light-quarks, namely the up, down, and strange quarks;

- the gluon;

- the large families of light mesonic particles made up of the light-quarks and gluons;

- the charm quark

- the $\mathrm{J} / \psi$ and $\psi^{\prime}$ resonances consisting of a charm and an anticharm quark; and

- the D mesons made up of a charm quark, a light-quark and gluons.

- the baryons composed of charmed and light quarks 
We refer to the physics of this large group of particles as tau, charm, light-quark and gluon physics.

Since the initial development of the Tau-Charm Factory concept our knowledge of tau, charm, light-quark and gluon physics has substantially increased. Also, another type of electronpositron collider, the B-Factory, has been developed and three such facilities have been or are being built.* Since the primary purpose of a $\mathrm{B}^{* *}$-Factory facility is the study of the physics of the heavy b-quark, the B-Factory operates in a narrow energy range near $10 \mathrm{GeV}$, compared to the 3-5 $\mathrm{GeV}$ energy range of the Tau-Charm Factory. However a B-Factory facility can be used for the study of many aspects of tau and charm physics and will accumulate large volumes of data in this respect.

A small international workshop on the 21st Century role of a Tau-Charm Factory in tau, charm, light-quark and gluon physics was held March 6-9, 1999, to review the physics capabilities and physics objectives of the Tau-Charm Factory concept. Participants came from North America, China, and Western Europe. The Workshop, held at the Stanford Linear Accelerator Center, had four goals:

(a) to review our present understanding of tau, charm, light-quark and gluon physics;

(b) to stimulate new ideas and experimental direction in tau, charm, light-quark and gluon physics;

(c) to summarize what we would like to learn, and might learn in these areas using a Tau-Charm Factory or a B-Factory or other experimental facility; and

(d) to consider the role, advantages, and disadvantages of using a Tau-Charm Factory facility to study tau, charm, light-quark and gluon physics, as compared to a B-Factory facility or other accelerator facility.

To achieve these goals the Workshop participants (Appendix) included many experts in tau, charm, light-quark and gluon physics; including those with experience in the B-Factory energy range as well as experimenters with experience in the Tau-Charm Factory energy range. Participants also included experts in the physics, design, construction and operation of electronpositron colliders and in the design, construction, and operation of particle detectors using the most

\footnotetext{
* We are here including the nearly completed B-factories at SLAC and KEK and the CESR facility at Cornell which has reached record luminosities and is being upgraded further.

** B mesons are combinations of b-quarks with other quarks.
} 
modern concepts. Viewgraphs and detailed papers given at the workshop are available at: http://www.slac.stanford.edu/grp/ee/TCP/TCPW.html

\section{Collider and Detector Design Status}

\section{a. Collider Design Status}

Although the primary purpose of the Workshop was to consider the particle physics capability and scope of a Tau-Charm Factory, there was a thorough presentation with extensive discussion on the design status and technical feasibility of a Tau-Charm Factory collider with an initial design luminosity of $10^{33} \mathrm{~cm}^{-2} \mathrm{~s}^{-1}$. The overall collider design as proposed by IHEP in the "BTCF feasibility study" was shown to be fully compatible with this luminosity and with the longitudinal polarization of either the electron beam or of both beams.

A number of collider design topics were discussed, subjects that should be studied to support an engineering design. One item is the influence of the Touschek Effect (intra-beam scattering) on backgrounds at the detector, beam lifetime and luminosity. There is a need for a comprehensive study of the beam-caused-backgrounds in the detector.

There was discussion of the beam polarization option. Single beam (electron) polarization requires incorporating a photoelectron gun of the type in use at the SLC; a straightforward addition. Polarizing both beams is a more extensive, but feasible, undertaking. There was discussion of the so-called monochromator option in the collider design, an option that would decrease the energy spread of the beam by an order of magnitude. The general opinion at the Workshop is that this option increases the complexity of the collider design and reduces the luminosity by an order of magnitude. Under these circumstances its contribution to the physics capability of the Tau-Charm Factory is expected to be small.

The summary judgment was reached that design and construction of a Tau-Charm Factory would be challenging but would be of difficulty comparable to that of a B-Factory.

The workshop discussed the potential of the BEPC machine if it were to be upgraded to the limit permitted by technology. It was judged that an increase in luminosity by a factor of five from the present peak luminosity at the $J / \psi$ energy could be obtained. While such upgrades might very well be valuable during the next few years when the BEPC can make further contributions, there remains a wide gap in terms of physics potential between such further upgrades and the expected 
performance of a Tau Charm Factory: the luminosity for the Tau Charm Factory is expected to be two orders of magnitude larger than that of the BEPC upgraded to its technical limit. If it were decided to pursue further upgrades, care must of course be taken that they do not require prolonged downtimes. Note that to benefit from such a further upgrade of the BEPC, the BES detector must also be upgraded. The workshop did not review costs of either the Tau-Charm Factory or of a regime of progressive upgrades of the BEPC, but made it clear that technically both or either could be accomplished.

\section{b. Detector Design Status}

There was no extensive effort at the Workshop to consider the detailed design of a detector for a Tau-Charm Factory. However there were several presentations and considerable discussion on detector parameters and technology. It was pointed out that the R\&D, design, and construction of a detector for a Tau-Charm Factory would provide an exciting opportunity for the use of new ideas and new technology in a large particle detector. Detectors to be used in the B-Factories and in the Large Hadron Collider are already constructed or in the course of construction. The design of detectors for the LHC is frozen. Therefore a Tau-Charm Factory detector would be the only new major detector effort initiated for several years. The initiation of a Tau-Charm Factory project would be very attractive to physicists and engineers interested in the R\&D and design of such a new large particle detector.

\section{Evaluation Criteria for Physics Capability of a Tau-Charm Factory}

\section{a. Evaluation criteria}

An important question addressed by the Workshop was: how does the physics community evaluate the physics capabilities of a proposed large experimental facility for basic science, such as a high energy physics accelerator, a synchrotron light source or a high flux neutron facility? Two criteria are considered in such an evaluation. Crucial is the evaluation of the amount of significant and fundamental physics research that can be accomplished using the proposed facility-the significant physics criterion. The second, and much more speculative, criterion is the major discovery potential criterion. The Workshop discussions on the definition and exposition of these criteria are summarized as follows: 
Significant physics contributions take a number of forms, for example:

- The physics results are of worldwide value and interest to other physicists in the field; for example, those who want a better understanding of the tau lepton need more precise measurements of the decay modes of the tau.

- The physics results are needed to augment other measurements. For example the comparison with theoretical predictions of very precise measurements of the magnetic moment of the muon requires precise measurements of the cross section for hadron production in the Tau Charm Factory energy range.

- The physics results contribute to the steady build up of more and more information about an elementary particle physics phenomenon. For example, the evidence for the existence of the gluon came from the accumulation of data on the directional distribution of mesons produced in electron-positron collisions.

The history of elementary particle physics teaches us that the major discovery potential criterion is much more a matter of judgment than the significant physics criterion. We mean by a major discovery, the physics result that changes drastically our understanding of particle physics. An example is the discovery of $\mathrm{CP}$ violation in the $\mathrm{K}$ meson decays.

The major discovery potential criterion must be addressed with caution since historically very rarely has such a potential been realized when new colliders and accelerators were initially proposed and constructed. To put it bluntly, most past high energy physics machines have been constructed for the wrong reasons. In fact in practice new accelerators and colliders have almost always met their projected significant physics objectives but even then their most important impact has turned out to be in areas not foreseen in the original proposals. Examples abound: SLAC was built with the main objective of extending past elastic electron scattering results but its most profound initial impact was through uncovering evidence of a point-like sub-structure of the nucleons; further SLAC served as injector to storage rings making profound and far-reaching discoveries. The Berkeley Bevatron was constructed with the specific objective to discover the anti-proton. This indeed was accomplished but the most profound impact was the discovery and exploitation of a very rich area of hadron spectroscopy. However sometimes the proposal for a new high energy facility extends hope that a specific major discovery will be made, but nature may or may not disappoint the experimenter. Consider the history of searches for the massive particles predicted by supersymmetric theory. One of the reasons for building the TEVATRON proton-anti 
proton collider and the LEP electron-positron collider was the potential for discovering supersymmetric particles. So far no such discovery has been made at either facility. If they do exist, their masses appear to be too large for detection. On the other hand, the proposals for both facilities met the significant physics criterion; they have indeed contributed immense amounts of significant physics results for example in accumulating data on the B quark and discovering the theoretically predicted top quark, and determining precise parameters for the electroweak interaction.

The workshop attempted to evaluate the major discovery potential of the planned experimental facility and in addition considered the breadth of the significant physics research that can be done, the possibilities for that research to lead to new ideas, and the possibilities for technological improvements and research expansions of the facility.

\section{b. Comparisons of the Tau Charm Factory with B-Factories}

Current results from the symmetric B-factory CESR and the projected capabilities of the soon to be completed asymmetric B-factories PEP II and KEK B were major considerations in the workshop discussions. While the major purpose of these facilities is to explore the physics of B mesons, particularly the expected CP violation physics in the B-system, substantial research in tau lepton physics and D meson physics can and will be carried out. Therefore in the following physics sections of this report there is repeated discussion of whether some of the physics that can be carried out at a Tau-Charm Factory is expected to be addressed equally well, or perhaps better, at a B-Factory.

In comparing the luminosities of a Tau-Charm Factory and a B-Factory we have used:

$$
\begin{aligned}
& \text { Design luminosity of Tau-Charm Factory }=10^{33} \mathrm{~cm}^{-2} \mathrm{~s}^{-1} \\
& \text { Design luminosity of B-Factory }=3 \times 10^{33} \mathrm{~cm}^{-2} \mathrm{~s}^{-1-}
\end{aligned}
$$

We have also assumed that as the Tau-Charm Factory and the B-Factories are upgraded in the future the ratio of luminosities of the Tau-Charm factory to that of the B-factory will be maintained approximately. 


\section{Glueball and Other Strong Interaction Physics}

\section{a. The $\mathrm{J} / \psi$ and $\psi^{\prime}$ charmonium resonances}

In the Tau-Charm Factory energy range, but not in the B-Factory energy range, there are two resonant states that are extremely important for the study of hadronic particles and quantum chromodynamics. As shown in Fig. 1 , these are the $\mathrm{J} / \psi$ resonance at $3.10 \mathrm{GeV}$ and the $\psi^{\prime}$ resonance at $3.69 \mathrm{GeV}$. Both are bound states of a charm quark and an anti-charm quark (charmonium). These resonances decay to a large variety of hadronic states, whose decays and properties allow the study of a tremendous amount of significant elementary particle physics. Preeminent among these studies is the possibility of finally answering the question of the existence of purely gluonic matter.

\section{b. Gluonic matter and glueballs}

All known hadronic particles consists of combinations of quarks and gluons; the gluons may be thought of as the particles that carry the strong force between the quarks, holding the particle together. But it has been known for decades that the possibility exists that there may be matter that consists purely of gluons. Such a particle is called a glueball. In spite of numerous searches and some indications of the existence of glueballs, we do not know that glueballs exist although there are candidate states which may be glueballs. A fully persuasive finding and identification of one or more glueballs would be a major discovery. It is without exaggeration to say that the Tau-Charm Factory could potentially discover not just one or two, but all the low-mass glueball states. Theory predicts the quantum numbers which a glueball may carry.

By far the best way to establish the existence of glueballs is to use the process

$$
\mathrm{J} / \psi \rightarrow \text { gluons }+ \text { photon }
$$

through a complete analysis of the spin and parity of candidate states. Such a study requires the production of a very large number of $\mathrm{J} / \psi$ events. Calculations at this Workshop put the required number between $10^{8}$, but more likely $10^{10}$ events! This can only be achieved at a Tau-Charm Factory. One reason for the required large number of $\mathrm{J} / \psi$ events is that it is probable that states exist that are quantum mechanical mixtures of a glueball and a conventional meson. The finding of such states would also be a significant physics result. 


\section{c. Other significant physics using the $\mathrm{J} / \psi$ and $\psi^{\prime}$}

As already noted a variety of significant elementary physics studies can be carried out using the $\mathrm{J} / \psi$ and $\psi^{\prime}$. We list a few examples

- The unraveling of puzzles related to the comparison of the properties of the $\mathrm{J} / \psi$ and $\psi^{\prime}$.

- The precise study of other charmonium states.

- The precise study of exotic $\mathrm{J} / \psi$ and $\psi^{\prime}$ decay modes to mesons and baryons.

- Searches for very rare decays of the $\mathrm{J} / \psi$, for example

$$
\mathrm{J} / \psi \rightarrow \mathrm{D}_{\mathrm{S}} \text { meson + lepton + neutrino. }
$$

\section{d. Two-virtual-photon physics}

In electron-positron collisions of the type

$$
\text { electron }+ \text { positron } \rightarrow \text { electron }+ \text { positron }+ \text { neutral meson, }
$$

the neutral meson is made by the annihilation of two virtual photons produced in the electronpositron collision. In the Tau-Charm Factory energy range, this process allows studies in depth of the properties of neutral mesons in the mass range of 0.3 to $3 \mathrm{GeV}$.

\section{Tau Lepton Physics}

\section{a. Present knowledge of tau lepton physics and the future program}

In the past decade a great deal of knowledge has been acquired about the tau lepton, the most massive member of the charged lepton family-electron, muon, and tau. Therefore we are now at the stage where further progress in tau physics depends upon obtaining very precise general measurements and in looking for tau physics areas where there might be a major discovery.

This tau physics program calls for the use of all known methods of studying the tau. With the near-future termination of physics research at the LEP electron-positron collider, the only facilities for studying tau physics are B-Factories, the Tau-Charm Factory and at a much lower level of precision, the existing or upgraded BEPC. In this section we report the views of the 
Workshop participants as to the future roles of these facilities in tau research. A brief summary is: (a) much significant tau physics research can be carried at both types of collider, (b) significant areas of tau physics research are better carried out at a Tau-Charm Factory, and (c) it is difficult to evaluate the major discovery potential for tau physics at either type of collider.

b. Tau threshold energy and preferred energy region for tau physics research at a Tau-Charm Factory

The minimum energy for producing tau lepton pairs is about $3.56 \mathrm{GeV}$, the tau threshold energy, as shown in Fig. 1. The best energy region for tau physics research at a Tau-Charm Factory is between the tau threshold energy and $3.67 \mathrm{GeV}$, an energy just below the $\psi^{\prime}$ resonance. In this energy region the only contamination of tau pair events comes from ordinary meson production and well understood purely electromagnetic processes. This is the tau preferred energy region and is unique to the tau-charm factory. Above $3.67 \mathrm{GeV}$ backgrounds from decays of charmed states grow rapidly.

\section{c. Tau studies using the tau threshold energy at a Tau-Charm Factory}

There are some areas of tau physics that are best studied immediately above the tau threshold energy at a Tau-Charm Factory. A significant physics example is the study of radiative decays of the tau such as

$$
\begin{aligned}
& \text { tau } \rightarrow \text { muon }+ \text { neutrino }+ \text { neutrino }+ \text { photon } \\
& \text { tau } \rightarrow \text { pion }+ \text { neutrino }+ \text { photon }
\end{aligned}
$$

At the tau threshold energy the photon can be shown to come from the tau decay of interest. Above the tau threshold energy the detected photon may come from the decay of the other tau of the tau pair or from the tau pair production process itself, thus contaminating the measurement.

Another example of the use of the tau threshold energy is a very precise measurement of the tau mass.

Any major discovery in tau physics, whether made first in a B-Factory or a Tau-Charm Factory, must be validated by examining the possible contamination through misidentification of non-tau events. One can test for such misidentification by operating the Tau-Charm Factory below tau threshold energy. If the discovery signal persists the effect is caused by background contamination. 


\section{d. Search for forbidden tau decays}

In the Standard Model, tau decay modes such as

$$
\begin{aligned}
& \text { tau } \rightarrow \text { muon }+ \text { photon } \\
& \text { tau } \rightarrow \text { electron }+ \text { photon }
\end{aligned}
$$

are expected to have extremely small branching fractions. Presently measured upper limits are somewhat larger than $10^{-6}$. Identifying one of these decay modes at a branching fraction in the range of $10^{-6}$ to $10^{-8}$ would be a major discovery in tau physics. The Tau-Charm Factory is much preferred for these studies because the tau threshold energy region allows much better understanding of possible contamination from other types of tau decays as well as from other backgrounds.

\section{f. Longitudinal polarization of the colliding beams}

A significant area of tau physics involves studying tau decay dependence on the spin direction of the tau. At present this can only be done statistically by observing the decay pattern of both members of the tau pair. It has long been recognized that these spin physics measurements could be made with better clarity and precision if the colliding electron beam or the colliding positron beam or both beams were longitudinally polarized. An additional advantage of using a polarized beam is that upon reversal of the polarization, current theory predicts a known change in the decay characteristics of the tau. Such a test provides unambiguous confirmation of the validity of the spin related decay measurements.

Designs now exist for manipulating and preserving the polarization of either or both beams if the beams are transversely polarized at injection. Polarizing the electron beam from the injector is relatively simple. Polarization of the positron beam would be possible but more complicated. Polarization of just the electron beam is sufficient for most physics purposes.

Longitudinally polarized beams are not at present included in the two-ring B-Factories PEP II and KEK B. Attempting to retrofit polarized beams into these machines may lead to technical and construction problems. Therefore polarization is likely to be a less effective physics tool in a B-Factory compared to a Tau-Charm Factory.

The enhanced power of a Tau-Charm Factory with polarized beams provides the kind of research breadth that can lead to a major discovery. For example as discussed next, longitudinally polarized beams will strengthen the search for $\mathrm{CP}$ violation in tau decay. 


\section{g. Search for CP violation in tau decay}

One of the most exciting areas in elementary particle physics is that certain processes change if the direction of time is reversed. Since we cannot reverse time, we use the equivalent simultaneous change of particle charge, $\mathrm{C}$, and particle parity, $\mathrm{P}$. If the behavior of a decay process changes under time reversal it will change under a $\mathrm{CP}$ change. Hence this phenomenon is called violation of $\mathrm{CP}$ invariance, or briefly $\mathrm{CP}$ violation. $\mathrm{CP}$ violation has been extensively studied in the $\mathrm{K}$ mesons and the chief purpose of the B-Factories is to search for it in B mesons and study it in detail. The $\mathrm{K}$ and $\mathrm{B}$ mesons are composed of quarks and at present $\mathrm{CP}$ violation is attributed to the properties of these quarks. But the outstanding question remains of whether the interaction of leptons can also exhibit $\mathrm{CP}$ violation.

The best way to search for $\mathrm{CP}$ violation in lepton decay is to examine certain decay processes of the tau. One pioneer search at moderate sensitivity with a null result has been carried out at $10 \mathrm{GeV}$ energy. Experimental limits on $\mathrm{CP}$ violation in tau decay are significant physics, finding CP violation in tau decay would be a major discovery. The tau physics community is at the beginning of an extensive research effort that will require the full power of both B-Factories and a Tau-Charm Factory. The use of longitudinally polarized beams will greatly increase the power and variety of the search methods. Thus the search for CP violation in tau decay provides significant physics and may lead to a major discovery in a Tau-Charm Factory with a polarized beam.

\section{h. Mass of the tau neutrino}

The tau neutrino is presumed to be produced in all conventional decays of the tau. By studying certain of these decays, in particular to multi-pion final states plus the tau-neutrino, the mass of the tau neutrino can in principle be determined. The best measurements put the upper limit on the tau neutrino mass between 20 and $30 \mathrm{MeV}$. The reduction of this limit on the tau neutrino mass is significant physics. The actual determination of the mass would be a major discovery. It is difficult to determine how far the search for the mass can go below the present 20-30 MeV upper limit, but it appears that one can probe down to several MeV at a Tau-Charm Factory. The consensus at the Workshop was that the corresponding search at a B-Factory could not probe below $10 \mathrm{MeV}$. 


\section{i. Purely-leptonic tau decays}

The study of the purely-leptonic decays of the tau

$$
\begin{aligned}
& \text { tau } \rightarrow \text { electron }+ \text { neutrino }+ \text { neutrino } \\
& \text { tau } \rightarrow \text { muon }+ \text { neutrino }+ \text { neutrino }
\end{aligned}
$$

is significant physics if these decays completely confirmed electroweak interaction theory of the Standard Model. A deviation from the Standard Model prediction would be a major discovery. It appears that the study of the purely-leptonic tau decays can be carried out at either a B-Factory or a Tau-Charm Factory.

\section{j. General study of semileptonic tau decays}

There was considerable discussion at the workshop on the relative merits of studying semileptonic tau decays such as

$$
\text { tau } \rightarrow \text { several mesons }+ \text { neutrino }
$$

at a Tau-Charm Factory as compared to a B-Factory. There was no consensus, the relative merits of each facility depend upon detailed calculations that have not been made.

\section{$\underline{\text { k. A general observation on Tau-physics at B factories }}$}

The Workshop recognized that the detector operating conditions and the physics program of a B-Factory might inhibit some studies of tau decays. For the next decade or more the physics programs of all B-Factories will be dedicated to studying $\mathrm{CP}$ violation and other phenomena in $\mathrm{B}$ meson decays at the highest obtainable luminosities. Then, for example, triggering on two-prong events that are only of interest for tau physics studies might be ignored. (Almost $70 \%$ of all tau pair production give rise to the two-prong topology.) On the other hand, the physics program of a TauCharm Factory will have a dedicated tau physics component. 


\section{D Meson Physics}

a. The importance of the production of pure D meson pairs at a Tau-Charm Factory

D mesons are produced, and their physics can be studied, in B-Factories and in antiprotonproton colliders such as the TEVATRON, as well as in a Tau-Charm Factory. However at the Tau-Charm Factory energy of $3.77 \mathrm{GeV}$, Fig. 1, electron-positron annihilations produce exactly one pair of $\mathrm{D}$ mesons. This can be a pair of neutral $\mathrm{D}$ mesons, $\mathrm{D}^{0}+$ anti- $\mathrm{D}^{0}$, or a pair of charged $\mathrm{D}$ mesons, $\mathrm{D}^{+}+\mathrm{D}^{-}$. At this $3.77 \mathrm{GeV}$ point, not only are the kinematic properties of the two $\mathrm{D}$ mesons well known, but also the quantum mechanical states of the two D mesons are restricted. The $3.77 \mathrm{GeV}$ energy point is called the $\psi^{\prime \prime}$ resonance.

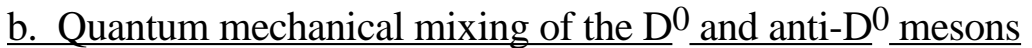

The quantum mechanical mixing of $\mathrm{D}^{0}$ and anti- $\mathrm{D}^{0}$ mesons is a significant area of quark physics. Such mixing occurs in other systems such as between the $\mathrm{K}^{0}$ and anti- $\mathrm{K}^{0}$. But present measurements of $\mathrm{D}^{0}$ and anti- $\mathrm{D}^{0}$ mixing are relatively primitive. Improved measurements can be made at B-Factories as well as at a Tau Charm Factory, but the use of D pair production at the $\psi^{\prime \prime}$ resonance at a Tau Charm Factory will give at least an order-of-magnitude improvement in the precision of the study of $\mathrm{D}^{0}$ and anti- $\mathrm{D}^{0}$ mixing.

The $\mathrm{D}^{0}$ and anti- $\mathrm{D}^{0}$ mixing phenomenon in principle allows the search for the violation of $\mathrm{CP}$ invariance in $\mathrm{D}$ meson decay. The discovery of $\mathrm{CP}$ violation in $\mathrm{D}$ decays would be a major discovery. Unfortunately present theory predicts that this phenomenon is much too small to be detected at a B-Factory, or at a Tau Charm Factory, or by any other known experimental method. Nevertheless, bold experimenters will search for this phenomenon, and their best chance, albeit small, is at a Tau-Charm Factory.

c. Rare and forbidden decays of D mesons and the use of the $\psi^{\prime \prime}$ resonance threshold

There is a large amount of significant physics involved in the study of the rare and forbidden decay modes of the D mesons. Examples of significant rare decay modes are

$$
\begin{aligned}
& \mathrm{D}^{+} \text {meson } \rightarrow \text { muon }+ \text { neutrino } \\
& \mathrm{D}^{+} \text {meson } \rightarrow \text { tau }+ \text { neutrino }
\end{aligned}
$$


These simple, but rare, decays provide basic parameters for $\mathrm{D}$ decay physics and test our ability to understand the physics of meson decay.

An example of a D decay forbidden by the Standard Model is

$$
\mathrm{D}^{0} \rightarrow \text { muon + electron }
$$

Evidence for such a decay would be a major discovery.

Studies of rare and forbidden D decays can be carried out at Tau-Charm Factories, BFactories, and to some extent at proton-proton colliders. However there is some advantage in using a Tau-Charm Factory with respect to the sureness of the measurement. In the study of rare or forbidden particle decays, there is always the worrisome possibility that the measurement has been contaminated by false events from other processes. In the case of $\mathrm{D}$ decays investigated at a Tau-Charm Factory this contamination can be directly measured by taking data below the $\psi^{\prime \prime}$ resonance. Then D meson pairs cannot be produced, and if there is a contamination from other types of events, that contamination can be evaluated. This energy threshold test can only be used at a Tau-Charm Factory.

\section{d. Improved measurements of known decays of D mesons}

There is a large amount of significant physics remaining to be done with respect to the general decays of $\mathrm{D}$ mesons, decays such as

$$
\begin{aligned}
& \mathrm{D} \rightarrow \pi \text { meson + lepton + neutrino } \\
& \mathrm{D} \rightarrow \mathrm{K} \text { meson + lepton + neutrino. }
\end{aligned}
$$

Generally these studies can be done equally well at a B-Factory or at a Tau Charm Factory. There are however a few special cases where a study would be most significant if carried out at a TauCharm Factory. For example, it is important to precisely measure the decay rate for

$$
\mathrm{D} \rightarrow \pi \text { meson }+\mathrm{K} \text { meson, }
$$

because this measurement is needed to absolutely standardize the other decay rates of the $\mathrm{D}$ meson and other hadrons. This measurement requires the use of a Tau Charm Factory. 


\section{e. $\mathrm{D}$ (and $\mathrm{D}_{\underline{s}}$ ) meson production in the 3.77 to $4.5 \mathrm{GeV}$ energy range}

It has been known for a long time that as the energy is increased above the $3.77 \mathrm{GeV} \psi^{\prime \prime}$ resonance, complex systems of higher states of $\mathrm{D}$ mesons are produced. A thorough investigation of this energy region requires a Tau-Charm Factory.

\section{Role of a Tau-Charm Factory in 21st Century Elementary Particle Physics}

\section{$\underline{\text { Conclusions }}$}

1.) Progress in the construction and successful initial operation of B-Factories has made us fully confident that building the collider and detector for a Tau-Charm Factory is feasible. The designers and builders of a Tau-Charm Factory will be able to learn continually from the experience and knowledge gained at B-Factories, and at the newly constructed DAPHNE PhiFactory, an electron-positron collider facility operating in the $1 \mathrm{GeV}$ energy range.

2.) The luminosity of a tau-charm factory is expected to be two orders of magnitude larger than that of the BEPC at its maximum upgrade potential.

3.) A Tau-Charm Factory is a unique facility for carrying out broad and significant research in gluon and light-quark physics.

4.) With respect to gluonic matter and the existence of glueballs, a Tau-Charm Factory offers a unique opportunity for a very important significant physics result.

5.) While tau and charm physics will continue to be studied at other facilities such BFactories, a Tau-Charm Factory provides competitive, and in some cases special, experimental conditions for carrying out significant physics in many areas of tau and charm physics.

6.) These special experimental conditions at a Tau-Charm Factory include: the use of the tau energy threshold for tau physics studies, the use of the $\psi^{\prime \prime}$ resonance for the study of D meson physics, and the naturalness of including a polarized beam facility in the initial construction of the collider. 
7.) The breadth of physics and the range of special experimental conditions inherent in a Tau-Charm Factory facility offer the possibility of major discoveries in areas such as $\mathrm{CP}$ violation and the lepton flavor non-conservation.

Above all:

8.) We look forward to exploring at the beginning of the 21 st Century those large parts of particle physics that we do not understand; therefore maximum range and variety in our experimental tools is crucial. A Tau-Charm Factory facility is different from a B-Factory facility or any other high energy physics facility. Therefore it amplifies the range and variety of experimental tools at a relatively moderate cost. 


\section{APPENDIX: WORKSHOP PARTICIPANTS}

\begin{tabular}{|c|c|}
\hline Rainer Bartoldus & University of Iowa bartoldu@slac.stanford.edu \\
\hline Michael Chanowitz & Lawrence Berkeley Laboratory mschanowitz@lbl.gov \\
\hline Jean Duboscq & Ohio State University jed@lns598.lns.cornell.edu \\
\hline William Dunwoodie & SLAC wmd@slac.stanford.edu \\
\hline Fred Gilman & Carnegie Mellon University gilman@cmphys.phys.cmu.edu \\
\hline JoAnne Hewett & SLAC hewett@slac.stanford.edu \\
\hline Colin Jessop & SLAC jessop@slac.stanford.edu \\
\hline Peter Kim & SLAC pck@slac.stanford.edu \\
\hline Jasper Kirkby & CERN jasper.kirkby@cern.ch \\
\hline Weiguo Li & IHEP Bejing liwg@sun.ihep.ac.cn \\
\hline Ted Liu & Lawrence Berkeley Laboratory thliu@lbl.gov \\
\hline Helmut Marsiske & SLAC masiske@slac.stanford.edu \\
\hline Wolfgang Panofsky & SLAC pief@slac.stanford.edu \\
\hline Martin Perl & SLAC martin@slac.stanford.edu \\
\hline Thomas Rizzo & SLAC rizzo@slac.stanford.edu \\
\hline Steven Robertson & University of Victoria stevenr@pp.phys.uvic.ca \\
\hline Vladimir Savinov & SLAC savinov@slac.stanford.edu \\
\hline Achim Stahl & University of Bonn stahl@pib1.physik.uni-bonn.de \\
\hline Ryszard Stroynowski & Southern Methodist University ryszard@mail.physics.smu.edu \\
\hline John Swain & Northeastern University John.Swain@ cern.ch \\
\hline Maury Tigner & Cornell University tigner@1ns62.lns.cornell.edu \\
\hline Walter Toki & Colorado State University toki@lamar.colostate.edu \\
\hline Paul Tsai & SLAC ystth@slac.stanford.edu \\
\hline Alan Weinstein & Caltech ajw@caltech.edu \\
\hline Xiaofan Zhou & SLAC zhouxf@slac.stanford.edu \\
\hline Yongsheng Zhu & IHEP Bejing zhuys@sun.ihep.ac.cn \\
\hline Bingsong Zou & IHEP Bejing zoubs@sun.ihep.ac.cn \\
\hline
\end{tabular}




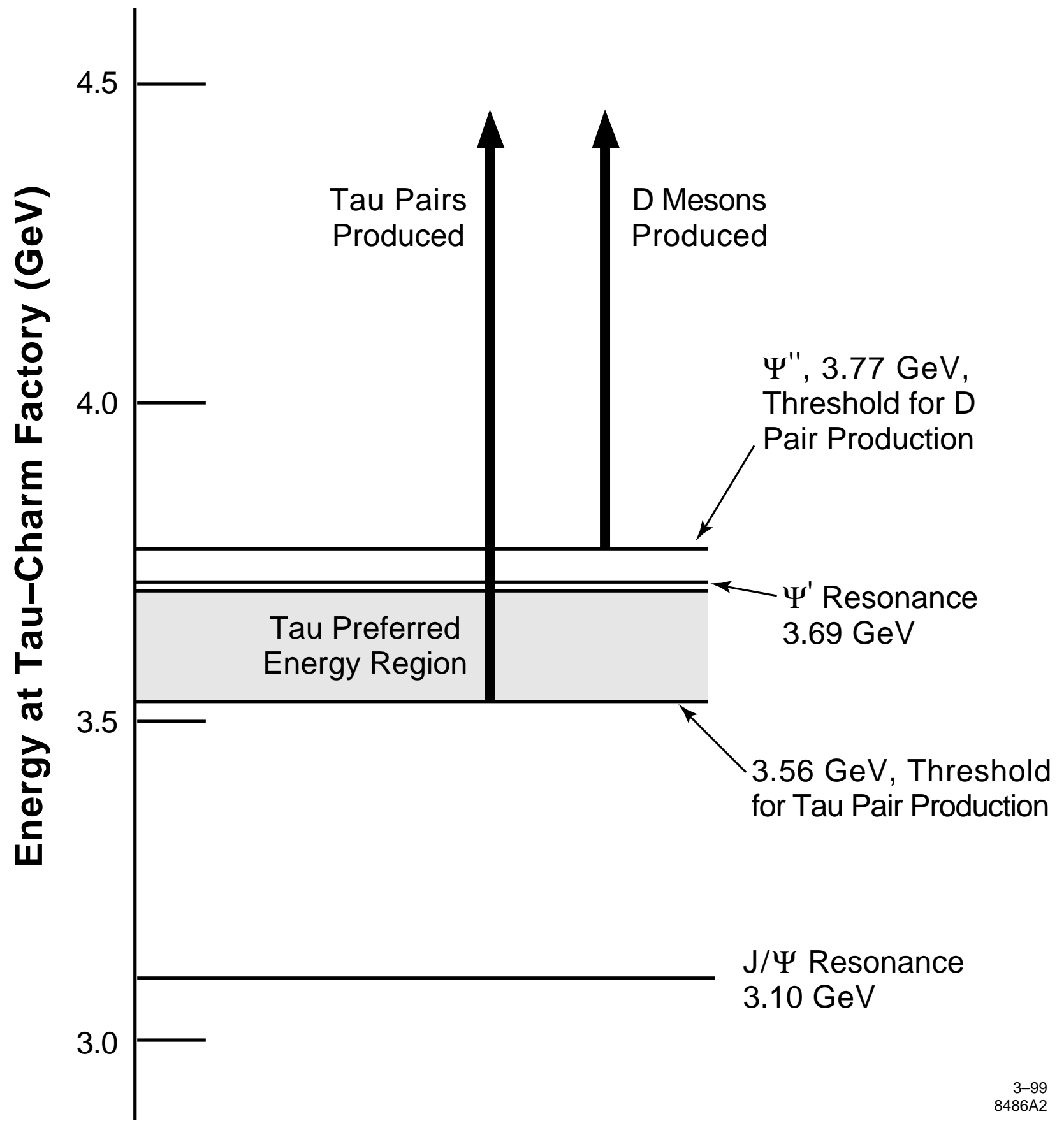

Figure 1. The special thresholds and energy regions used in a Tau-Charm Factory. 